\title{
Non-Quadratic Gauge Fixing and Global Gauge Invariance in the Effective Action
}

\author{
F. T. Brandt ${ }^{a}$ and D. G. C. McKeon ${ }^{b}$ \\ ${ }^{a}$ Instituto de Física, Universidade de São Paulo, São Paulo, SP 05315-970, Brazil and \\ ${ }^{b}$ Department of Applied Mathematics, University of Western Ontario, London, ON N6A 5Br, Canada
}

\begin{abstract}
The possibility of having a gauge fixing term in the effective Lagrangian that is not a quadratic expression has been explored in spin-two theories so as to have a propagator that is both traceless and transverse. We first show how this same approach can be used in spontaneously broken gauge theories as an alternate to the 't Hooft gauge fixing which avoids terms quadratic in the scalar fields. This "non-quadratic" gauge fixing in the effective action results in there being two complex Fermionic and one real Bosonic ghost fields. A global gauge invariance involving a Fermionic gauge parameter, analogous to the usual BRST invariance, is present in this effective action.
\end{abstract}

PACS numbers: $11.15 .-\mathrm{q}$

\section{INTRODUCTION}

The path integral quantization of gauge theories involves having to choose a gauge fixing condition that results in a "gauge fixing" term supplementing the classical Lagrangian. A further "ghost action" involving a complex Fermionic ghost field is also required in order to cancel the contribution of non-physical degrees of freedom in radiative process [1, 2, 3, 4]. One of the most remarkable outcomes of this conventional gauge fixing is that the full effective action possesses a global gauge invariance with a Fermionic gauge parameter known as "BRST invariance" [5, 6,6$]$.

In Ref. [8] it is pointed out how this procedure cannot be applied in the case of a spin-two gauge theory so as to have a spin-two propagator that is both traceless and transverse (TT). This difficult can be circumvented by modifying the "Faddeev-Popov" procedure so that a "gauge fixing" term appears in the effective Lagrangian that is not quadratic in some gauge fixing condition. In the next section we review this procedure and provide a second illustration of how it works in a $U(1)$ model in which there is spontaneous symmetry breaking.

The gauge fixing term will still have the advantage of the 't Hooft gauge [9, 10], which eliminates the terms bilinear in the gauge and scalar fields (which would appear if a Feynman gauge were used [1] ), and has the additional advantage of eliminating gauge dependent mass terms for the scalars which arise in the 't Hooft gauge. The nature of the ghost fields which arise with this gauge fixing is then discussed; it is shown that two Fermionic and one Bosonic ghost arise.

Having illustrated how non-quadratic gauge fixing terms can be incorporated into the effective action, we now show how this effective action possess a global gauge invariance which is a generalization of the BRST invariance of the usual effective action. Both the Fermionic and Bosonic ghosts participate in this transformation, with the unusual feature that the transformation of the Fermionic ghost is non-local.

\section{NON-QUADRATIC GAUGE FIXING}

We consider a vector gauge field interacting with a complex scalar field with classical Lagrangian

$$
\begin{aligned}
\mathcal{L}_{C l} & =-\left(\partial_{\mu}+i e V_{\mu}\right) \phi^{\star}\left(\partial_{\mu}-i e V_{\mu}\right) \phi \\
& -\frac{1}{4}\left(\partial_{\mu} V_{\nu}-\partial_{\nu} V_{\mu}\right)^{2}-m^{2} \phi^{\star} \phi-\lambda\left(\phi^{\star} \phi\right)^{2} .
\end{aligned}
$$

If $m^{2}<0$, spontaneous symmetry breakdown occurs, and if $f$ is the vacuum expectation value of $\phi$, so that

$$
\sqrt{2} \phi=f+h
$$

where $h$ is the quantum fluctuation about $\mathcal{C}$, then the model of (2.1) develops a contribution that is bilinear in $V_{\mu}$ and $h$. If the usual Feynman gauge fixing Lagrangian $-\frac{1}{2 \alpha}(\partial \cdot V)^{2}$ is used, these terms result in a mixed $\left\langle h V_{\mu}\right\rangle$ propagator which complicates the computation of radiative effects. In order to eliminate such terms, 't Hooft suggested using a modified gauge fixing Lagrangian 9, 10]

$$
\mathcal{L}^{(1)}=-\frac{1}{2 \alpha}\left[\partial \cdot V+\frac{i e \alpha}{2}\left(f^{\star} h-f h^{\star}\right)\right]^{2} ;
$$

in the sum of Eqs. (2.1) and (2.3) these cross terms cancel provided $f$ is a constant. The same advantage arises if one were to use the non-quadratic gauge fixing Lagrangian

$$
\mathcal{L}^{(2)}=-\frac{1}{2 \alpha}[\partial \cdot V]\left[\partial \cdot V+i e \alpha\left(f^{\star} h-f h^{\star}\right)\right]
$$

without the introduction of terms quadratic in $h, h^{\star}$

Incorporation of the gauge fixing term of Eq. (2.3) in the effective Lagrangian when using the path integral quantization involves the Faddeev-Popov procedure [3, 12], which must be extended in order to accommodate the gauge fixing of Eq. (2.4) [8]. We will briefly review this modification.

The features of the path integral in which we are interested can be illustrated by considering the integral over 
the components of an $n$-dimensional vector $\vec{h}$

$$
Z=\int \mathrm{d} \vec{h} \exp \left(-\vec{h}^{T} M_{\sim} \vec{h}\right)=\frac{\pi^{\frac{n}{2}}}{(\operatorname{det} M)^{\frac{1}{2}}}
$$

If $\underset{\sim}{M}$ is an $n \times n$ matrix such that $\underset{\sim}{M} \underset{\sim}{A \vec{\theta}})=0$ where $\vec{\theta}$ is an arbitrary vector then the integral in Eq. (2.5) is undefined. In order to excise the vanishing eigenvalue of $M$, we insert three factors of " 1 " into (2.5)

$$
\begin{aligned}
& 1=\int \mathrm{d} \vec{\theta}_{1} \delta\left(\underset{\sim}{F}\left(\vec{h}+\alpha \underset{\sim}{A \vec{\theta}_{1}}\right)-\vec{p}\right) \operatorname{det}(\alpha \underset{\sim}{F} \underset{\sim}{A}) \\
& 1=\int \mathrm{d} \vec{\theta}_{2} \delta\left(G\left(\vec{h}+\alpha \underset{\sim}{A \vec{\theta}_{2}}\right)-\vec{p}\right) \operatorname{det}(\alpha \underset{\sim}{A})
\end{aligned}
$$

and

$$
1=(\alpha \pi)^{-n} \int \mathrm{d} \vec{p} \mathrm{~d} \vec{q} \exp \left(-\frac{1}{\alpha} \vec{p}^{T} \underset{\sim}{N} \vec{q}\right) \operatorname{det} \underset{\sim}{N} .
$$

The shift

$$
\vec{h} \rightarrow \vec{h}-\alpha \underset{\sim}{A} \vec{\theta}_{1}
$$

leads to (after integrating out the $\vec{p}$ and $\vec{q}$ variables)

$$
\begin{aligned}
& Z=\left(\frac{\alpha}{\pi}\right)^{n} \int \mathrm{d} \vec{\theta}_{1} \int \mathrm{d} \vec{\theta} \int \mathrm{d} \vec{h} \operatorname{det}(\underset{\sim}{F} \underset{\sim}{A}) \operatorname{det}(\underset{\sim}{G A}) \\
& \times \operatorname{det}(\underset{\sim}{N}) \exp \left[-\vec{h}^{T}\left(\underset{\sim}{M}+\frac{1}{\alpha} \underset{\sim}{F^{T}} \underset{\sim}{N G}\right) \vec{\sim}\right. \\
& \left.-\vec{h}^{T} \underset{\sim}{F^{T}} \underset{\sim}{N G} \underset{\sim}{A \theta}\right]
\end{aligned}
$$

where $\vec{\theta}=\vec{\theta}_{2}-\vec{\theta}_{1}$. One could shift $\vec{h}$ to remove the "cross term" in $\vec{h}$ and $\vec{\theta}$ but this shift would not be a "gauge transformation" of the form of (2.7).

The determinants in Eq. (2.8) can be exponentiated using the standard Berezin integral

$$
\operatorname{det} \underset{\sim}{B}=\int \mathrm{d} \vec{c} \mathrm{~d} \overrightarrow{\vec{c}} \exp \left(-\overrightarrow{\vec{c}}^{T} \underset{\sim}{B} \vec{c}\right)
$$

where $\vec{c}, \vec{c}$ are Grassmann vectors; the first two determinants in Eq. (2.8) lead to "Faddeev-Popov" like ghosts and the third to a "Nielsen-Kallosh" ghosts [13, 14] which we will subsequently ignore, taking $\underset{\sim}{N}=$ const.. The field $\vec{\theta}$ is a "Bosonic" ghost. The integral over $\vec{\theta}_{1}$ in Eq. (2.8) parametrizes the divergence in Eq. (2.5) and can be absorbed into the normalization of $Z$.

In the model of Eq. (2.1), we make the identification

$$
\vec{h}=\left(V_{\mu}, h, h^{\star}\right)^{T}
$$

so that

$$
\underset{\sim}{A}=\left(\partial_{\mu}, i e(f+h),-i e\left(f^{\star}+h^{\star}\right)\right)^{T} .
$$

From Eqs. (2.4), (2.6) and (2.7) $\vec{\theta}$ is a scalar and

$$
\begin{aligned}
& \underset{\sim}{F}=\left(\partial_{\mu}, 0,0\right), \\
& \underset{\sim}{G}=\left(\partial_{\mu}, i e \alpha f^{\star},-i e \alpha f\right), \\
& \underset{\sim}{N}=\frac{1}{2}
\end{aligned}
$$

so that the ghost actions are

$$
\mathcal{L}_{b}=\bar{b}\left(\partial^{2}\right) b
$$

and

$$
\mathcal{L}_{c}=\bar{c}\left[\partial^{2}-2 \alpha e^{2} f^{\star} f-\alpha e^{2}\left(f^{\star} h+f h^{\star}\right)\right] c
$$

and the argument of the exponential in Eq. (2.8) is

$\mathcal{L}_{T}=\mathcal{L}_{C l}+\mathcal{L}_{g f}^{(2)}-V^{\mu} \partial_{\mu}\left[\partial^{2}-\alpha e^{2}\left(2 f^{\star} f+f^{\star} h+f h^{\star}\right)\right] \theta$

We now examine the global invariance possessed by the effective Lagrangian

$$
\mathcal{L}_{\text {eff }}=\mathcal{L}_{T}+\mathcal{L}_{b}+\mathcal{L}_{c} .
$$

\section{GLOBAL GAUGE INVARIANCE}

It is actually simpler to discuss the effective action before the fields $\vec{p}$ and $\vec{q}$ in Eq. (2.6c) are eliminated and without making the shift of Eq. (2.7) so that

$$
\begin{aligned}
& \mathcal{L}_{\text {eff }}=-\vec{h}^{T} \underset{\sim}{M} \vec{h}+\frac{1}{\alpha}\left[\vec{p}^{T} \underset{\sim}{\underset{h}{F}}\left(\vec{h}+\alpha \underset{\sim}{A} \vec{\theta}_{1}\right)\right. \\
& \left.+\vec{q}^{T} \underset{\sim}{G}\left(\vec{h}+\alpha \underset{\sim}{A \theta_{2}}\right)+\vec{p}^{T}{\underset{\sim}{N}}^{-1} \vec{q}\right] \\
& +\underset{\sim}{\vec{b}} \underset{\sim}{A \vec{b}}+\overrightarrow{\vec{c}} \underset{\sim}{A} \underset{\sim}{A \vec{c}}+\overrightarrow{\vec{k}} \underset{\sim}{F} \underset{\sim}{A \vec{k}}
\end{aligned}
$$

Following the usual BRST transformation, we begin with

$$
\begin{aligned}
\delta \vec{h} & =\underset{\sim}{A(\xi \vec{c}+\zeta \vec{d}) \epsilon} \\
\delta \vec{p} & =\delta \vec{q}=0=\delta \overrightarrow{\vec{k}}=\delta \vec{k}
\end{aligned}
$$

where $\epsilon$ is a Grassmann constant and $\xi$ and $\zeta$ are ordinary constants. If now the ghost fields are also varied then the effective Lagrangian also undergoes the change

$$
\begin{aligned}
& \delta \mathcal{L}_{e f f}=\frac{1}{\alpha}\left[\vec{p}^{T} \underset{\sim}{F}\left(\delta \vec{h}+\alpha \underset{\sim}{A} \delta \vec{\theta}_{1}+\alpha \underset{\sim}{A}, \vec{\theta}_{1} \delta h_{l}\right)\right. \\
& \left.+\vec{q}^{T} G\left(\delta \vec{h}+\alpha \underset{\sim}{A} \delta \vec{\theta}_{2}+\alpha \underset{\sim}{A}, \vec{\theta}_{2} \delta h_{l}\right)\right] \\
& +\delta \underset{\sim}{\vec{b}} \underset{\sim}{A} \underset{\sim}{A b}+\underset{\sim}{\vec{b}} \underset{\sim}{A_{\sim}} A_{l} \delta h_{l} \vec{b}+\underset{\sim}{\vec{b}} \underset{\sim}{A} \underset{\sim}{A} \delta \vec{b} \\
& +\delta \overrightarrow{\vec{c}}{\underset{\sim}{A}}_{\sim}^{A \vec{c}}+\overrightarrow{\vec{c}} \underbrace{}_{\sim} A_{\sim}, l h_{l} \vec{c}+\overrightarrow{\vec{c}} \sim_{\sim}^{A} \underset{\sim}{A} \delta \vec{c}
\end{aligned}
$$

We have used the fact that the classical Lagrangian is unaltered under the transformation of Eqs. (3.2) and 
assumed that $\underset{\sim}{N}$ is an invariant. In order to ensure that in Eq. (3.3) $\delta \mathcal{L}_{\text {eff }}=0$, Eq. (3.2) must be supplemented by

$$
\begin{gathered}
\delta \vec{\theta}_{1}=-\frac{\zeta}{\alpha} \vec{c} \epsilon, \\
\delta \vec{\theta}_{2}=-\frac{\xi}{\alpha} \vec{b} \epsilon, \\
\delta b_{i}=\frac{1}{2} \xi f_{a b ; i} b_{a} b_{b} \epsilon, \\
\delta c_{i}=\frac{1}{2} \zeta f_{a b ; i} c_{a} c_{b} \epsilon,
\end{gathered}
$$

$$
\begin{aligned}
& \delta \bar{b}_{i}-\xi p_{i} \epsilon-\alpha \xi\left(\vec{p}^{T} \underset{\sim}{F} \underset{\sim}{A}, \vec{\theta}_{1}\right)\left(\underset{\sim}{A \Lambda^{-1}}\right)_{l i} \epsilon \\
& -\alpha \xi\left(\vec{q}^{T} \underset{\sim}{G \underset{\sim}{A}, \vec{\theta}_{2}}\right)\left(\underset{\sim}{A \Lambda^{-1}}\right)_{l i} \epsilon \\
& -\xi\left(\vec{c}^{T}{\underset{\sim}{A}}_{\sim, l} \vec{c}\right)\left(\underset{\sim}{A \Lambda^{-1}}\right)_{l i} \epsilon=0, \\
& \delta \bar{c}_{i}-\zeta q_{i} \epsilon-\alpha \zeta\left(\vec{q}^{T}{\underset{\sim}{A} \sim_{,}, \vec{\theta}_{2}}\right)\left(\underset{\sim}{A \Sigma_{\sim}^{-1}}\right)_{l i} \epsilon \\
& -\alpha \zeta\left(\vec{p}^{T} \underset{\sim}{F} \underset{\sim}{A}, \vec{\theta}_{1}\right)\left(\underset{\sim}{A \Sigma^{-1}}\right)_{l i} \epsilon \\
& -\zeta\left(\vec{b}^{T} \underset{\sim}{F} \underset{\sim}{A}, \vec{b}\right)\left(\underset{\sim}{A \Sigma_{\sim}^{-1}}\right)_{l i} \epsilon=0, \\
& \left(\stackrel{\sim}{\perp} \underset{\sim}{F} \underset{\sim}{A}, \quad \underset{\sim}{\sum} \equiv \underset{\sim}{G A}\right) .
\end{aligned}
$$

In Eqs. (3.5a) and (3.5b) we have used the identity

$$
A_{a b, c} A_{c d}-A_{a d, c} A_{c b}=f_{b d ; c} A_{a c} .
$$

This follows from the fact that the commutator of two gauge transformations must be a gauge transformation.

If the shift of Eq. (2.7) were to occur, then in Eqs. (3.1), (3.2), (3.3), (3.4), (3.5) and (3.6) one sets $\vec{\theta}_{1}=\zeta=$ $0, \vec{\theta}_{2}=\theta$.

Because of the presence of the operators $\underset{\sim}{\Lambda},{\underset{\sim}{\Sigma}}^{-1}$ in Eqs. (3.6a) and (3.6b) these transformations are nonlocal. With the identifications of Eqs. (2.12), the transformations of Eqs. (3.4), (3.5) and (3.6) become

$$
\begin{aligned}
\delta \theta_{1} & =-\frac{\zeta}{\alpha} c \epsilon, \quad \delta \theta_{2}=-\frac{\xi}{\alpha} b \epsilon, \\
\delta b & =\delta c=0, \\
\delta \bar{b} & =\xi p \epsilon-i \alpha e^{3} \partial^{-2}\left[\alpha \xi q\left(f^{\star} h-f h^{\star}\right) \theta_{2}+\bar{c}\left(f^{\star} h-f h^{\star}\right) c\right] \epsilon, \\
\delta \bar{c} & =\xi q \epsilon-i \alpha e^{3}\left[\partial^{2}-2 \alpha e^{2} f^{\star} f-\alpha\left(f^{\star} h+f h^{\star}\right)\right]^{-1}\left[\alpha \zeta p\left(f^{\star} h-f h^{\star}\right) \theta_{1}+\bar{b}\left(f^{\star} h-f h^{\star}\right) b\right] \epsilon .
\end{aligned}
$$

These transformations can be used to find relationships between Green's functions in much the same way that the usual BRST transformations can be employed.

\section{DISCUSSION}

We have demonstrated that non-quadratic gauge fixing terms can be used in spontaneously broken theories and that these give rise to two Fermionic and one Bosonic ghosts. The full effective action possess a global gauge invariance with a constant Grassmann gauge parameter. This ensures that the transformation is a cohomology.

The existence of a global gauge invariance in the effective action introduced above may make it possible to devise a quantization procedure similar to the "BRST quantization" procedure of Refs. [15, 16]. This would guarantee that the theory is unitary and ghost-free, an interesting problem especially if one wants to use the TTgauge discussed in Ref. [8].

\section{Acknowledgments}

F. T. Brandt would like to thank Fapesp and CNPq for financial support. D. G. C. McKeon would like to thank R. MacLeod for a helpful suggestion. 
[1] R. P. Feynman, Acta Phys. Polon. 24, 697 (1963).

[2] B. S. DeWitt, Phys. Rev. 160, 1113 (1967).

[3] L. D. Faddeev and V. N. Popov, Phys. Lett. B25, 29 (1967).

[4] S. Mandelstam, Phys. Rev. 175, 1580 (1968).

[5] I. V. Tyutin (1975), 0812.0580.

[6] M. Z. Iofa and I. V. Tyutin, Teor. Mat. Fiz. 27, 38 (1976).

[7] C. Becchi, A. Rouet, and R. Stora, Annals Phys. 98, 287 (1976).

[8] F. T. Brandt, J. Frenkel, and D. G. C. McKeon, Phys. Rev. D76, 105029 (2007), 0707.2590.
[9] G. 't Hooft, Nucl. Phys. B35, 167 (1971).

[10] K. Fujikawa, B. W. Lee, and A. I. Sanda, Phys. Rev. D6, 2923 (1972).

[11] B. W. Lee, Phys. Rev. D5, 823 (1972).

[12] G. 't Hooft, Nucl. Phys. B33, 173 (1971).

[13] N. K. Nielsen, Nucl. Phys. B140, 499 (1978).

[14] R. E. Kallosh, Nucl. Phys. B141, 141 (1978).

[15] G. Curci and R. Ferrari, Nuovo Cim. A35, 273 (1976).

[16] T. Kugo and I. Ojima, Prog. Theor. Phys. 60, 1869 (1978). 